\section{$\underset{\substack{\text { hommes } \\ \text { \& migrations }}}{ }$}

\section{Hommes \& migrations}

Revue française de référence sur les dynamiques

migratoires

\section{$1322 \mid 2018$}

Exposer les migrations

\title{
Sous le signe d'un mois de mai
}

\section{Mustapha Harzoune}

\section{OpenEdition \\ Journals}

\section{Édition électronique}

URL : https://journals.openedition.org/hommesmigrations/6865

DOI : 10.4000/hommesmigrations.6865

ISSN : 2262-3353

\section{Éditeur}

Musée national de l'histoire de l'immigration

\section{Édition imprimée}

Date de publication : 1 juillet 2018

Pagination : 197-201

ISBN : 978-2-919040-42-1

ISSN : $1142-852 X$

\section{Référence électronique}

Mustapha Harzoune, «Sous le signe d'un mois de mai », Hommes \& migrations [En ligne], 1322 | 2018, mis en ligne le 01 juillet 2018, consulté le 21 janvier 2022. URL : http://journals.openedition.org/ hommesmigrations/6865; DOI : https://doi.org/10.4000/hommesmigrations.6865 


\title{
SOUS LE SIGNE D'UN MOIS DE MAI
}

\author{
Par MUSTAPHA HARZOUNE.
}

T andis que mai s'ébrouait sous les premiers rayons de soleil, la société soufflait 50 bougies d'une révolution printanière. La tronche lumineuse et les yeux bleus d'un juif allemand, née à Montauban de parents exilés, lui-même expulsé fissa en 68 et "naturalisé » en 2015, continuait d'incarner cette page enthousiaste du roman national. Si la France n'est pas spontanément une terre d'accueil, son histoire, souvent, est portée par quelques « bâtards »-franco-allemand en l'occurrence. Le 30 mai 1968 , certains manifestants progaullistes ne s'y étaient pas trompés en scandant "le vieux mot d'ordre de l'extrême droite", "la France aux Français". Ils se doutaient que les "racines" françaises se régénèrent sous les semelles de vent de quelques métèques. Quant à imaginer qu'elles pousseraient dans le béton (voir l'exposition de Kader Attia au Mac Val), on eut été, quinze ans avant une marche en babouches et charentaises, en pleine science-fiction.

50 ans donc. Et les passions continuent de bruire. Des espérances et des valeurs intactes pour les uns. Les mêmes répulsions pour d'autres. La haine contre une jeunesse trop libertaire et déjà cosmopolite et des ouvriers par trop mobilisés (lire I'historienne Ludivine Bantigny). La fiction d'une "France aux Français » imagine une France momifiée et des Français immaculés de cette "prétendue pureté d'un sang mille fois mêlé depuis les Celtes" (Blas de Roblès). Cette France, phobique des métèques, bouffie d'orgueil et défigurée par une haine ulcérogène, préfère Maurras à Hugo, Le Pen à Cohn-Bendit, son riquiqui pré-carré à la vaste Europe.
Le pays a changé : on y vit onze ans de plus, on y enfante hors mariage et batifole à l'ombre d'un Pacs. Les Françaises retardent l'âge de la maternité. 68 est passé par là. Mais, si sous l'ennui gaullien couvait la révolte, sous le soleil de mai les vieilles lunes ne désarmaient pas. Les « Trente glorieuses » allaient être remisées. Et les mains du travailleur immigré - chantées par Zebda, évoquées par Ahmed Kalouaz - itou. La France passera du « travailleur immigré au migrant », écrit Agnès Laurent (L'Express, 3 janvier 2018). En 1974, Giscard, croyant fermer les frontières, inaugure des décennies de politiques où l'anémique carotte (de l'intégration) masque mal le gros bâton (de l'exclusion). Sur le sujet, de "sa suffisance " à "Jupiter ", l'imagination n'a plus jamais été au pouvoir. Comme l'écrivait Charles-Louis Philippe, "les mots sont les fantômes des imaginations malades, au-dessus desquels il y a la vie qu'il faut vivre sans penser aux mots ". Alors, de 1968 à 2014, les immigrés passent de 3 millions (6,6 \% de la population) à 5,9 millions (8,9\%), et les étrangers de 2,6 millions $(5,4 \%)$ à 4,1 millions (6,2\%). La France est bien dans le monde, et le monde en France : "La société n'en finit plus de se métisser. Et, pour une partie de celle-ci, de se crisper ", écrit Agnès Laurent.

\section{L'imagination au pouvoir !}

Si la royale reine d'Angleterre se déride, ne peut-on imaginer que la France républicaine cesse de se turlupiner ? Car les Windsor ont accepté de prendre des couleurs, grâce au mariage d'un prince et d'une 


\section{KIOSQUE}

ci-devant actrice. Megan Markle, métisse américaine (une Noire selon l'entendement états-unien), compte par sa mère un quadrisaïeul qui fut esclave jusqu'en 1865. Sans bêcher, Megan Markle n'est pas Othello pour « renflamme[r] nos courages éteints ». D'ailleurs, l'arrivée d'un métis (un Noir) à la Maison blanche n'a pas levé « l'obstacle éternel au mélange des races " comme l'annonçait Gustave de Beaumont en... 1835. "Sois qui tu veux, pur ou métis. Une fois riche, tu deviendras le maitre " enseignait, il y a quelques mille ans, l'immense Abû I-'Alâ' al-Maari, préfigurant là les analyses de Walter Benn Michaels (La diversité contre l'égalité, Raisons d'agir, 2009).

\section{Faites l'amour pas la guerre !}

Si «le poisson pourri par la tête », c'est sûrement par son « corps » que change la société. «La beauté est dans la rue" scandait-on en 68. Alors, les 33800 mariages mixtes célébrés en France en 2015 , soit $14 \%$ des 236000 mariages ( $6 \%$ en 1950) - plus les 42000 mariages à l'étranger, les unions libres et autres Pacs - feront plus pour « renflamme[r] nos courages éteints » que l'hymen monarchique et surmédiatisé.

"Nous devons découvrir le pouvoir de l'amour, le pouvoir de rédemption de l'amour, et ainsi, nous ferons de ce monde un nouveau monde " disait le révérend Michael Bruce Curry lors du fameux mariage. La citation, empruntée à Martin Luther King, provoqua quelques «regards médusés de certains membres de la couronne britannique" (Le Monde, 19 mai). Aux Étonnants voyageurs de Saint-Malo, Daniel Cohn-Bendit disait - à sa façon - la même chose sur le dispositif Erasmus "qui permet aux jeunes Européens d'étudier hors de leur pays ": "Imaginez, si, surle million d'entre eux, 300000 se rencontrent et fondent une famille. De quelle nationalité seront les enfants? C'est simple, ils seront Européens!"C'est ainsi que l'on change la société, reprenant un vieux slogan pacifiste qui a fait florès en 68 : "Faites l'amour, pas la guerre". ” (Ouest-France, 19 mai).

\section{Cours, camarade, le vieux monde est derrière toi}

Le vieux monde? Des politiques migratoires inefficaces et dangereuses ; le migrant pensé comme un coût et non comme une ressource ; un envahisseur au lieu d'une promesse, une menace plutôt qu'un pari. Le vieux monde? Une gestion des mobilités - "flux", «vague », "pression »! - qui relève de la mécanique des fluides versus une approche en réseaux ; le chacun pour soi patriotard plutôt qu'une coordination internationale ; des politiques de la charité plutôt que le dynamisme de l'échange ; la hiérarchie des différences versus le partage du commun... Pourtant, selon la Banque mondiale "les envois de fonds [des migrants] officiellement enregistrés vers les pays à revenu faible et intermédiaire ont atteint 466 milliards de dollars en 2017 ». Trois fois plus que les 146,6 milliards de dollars de l'aide publique (Organisation de coopération et de développement économiques, OCDE). Au pays, ces envois permettent de scolariser les enfants, de se soigner ou de simplement manger. Et peut-être d'éviter... de partir. Ces «migrants » pourraient faire plus et mieux si les conditions politiques le permettaient, plus et mieux si l'argent transféré n'était pas taxé à hauteur de $7,45 \%$ pour 200 dollars et même à un coût moyen de $9,8 \%$ pour les transferts vers l'Afrique subsaharienne! Plus et mieux si, selon la Banque mondiale, "plusieurs pays à revenu élevé " n'envisageaient pas « d'imposer les envois de fonds, en partie pour augmenter leurs recettes et en partie pour dissuader les migrants sans papiers " (l'économiste.com, 25 avril, quotidien marocain). Le vieux monde est-il seulement à la traîne ? N'est-il pas en train de crapahuter devant ? Il est là, cherchant à déplacer ses frontières aussi loin que possible, comme le montre Virginie Guiraudon (CNRS) avec la création par la France d'un " hotspot " à Agadez au Niger (The Conversation, 22 mai). Ce hotspot "fait partie d'une politique de 
"contrôle à distance" mise en place dans les années 1990 pour décourager l'arrivée des migrants sur le sol européen. (...) II réduit ainsi la migration à un "problème" dont la solution est une question de logistique ". "Au final, écrit l'auteure, un centre de "hotspot" français à Agadez n'est pas tant une solution logistique que la quintessence de l'attitude des États membres de l'UE vis-à-vis de l'Afrique: condescendance diplomatique, collaboration avec des régimes dictatoriaux et méconnaissance des acteurs locaux."

Le vieux monde ressemble à cette extrême droite va-t-en-guerre qui fanfaronne au col de l'Échelle (Huffington Post, 21 avril), débarque aux Étonnants voyageurs (Ouest-France, 20 mai), multiplie les actions violentes et les " happenings », contamine le net via des «sites de désinformation [qui] se présentent également par un jeu de permutation comme des sites alternatifs, de "ré-information" (Stéphane François, École pratique des hautes études, The Conversation, 28 mars et 9 avril). En 1968, pour ouvrir les yeux, il fallait fermer la télé. En 2018, ce sont ces sites qu'il faudrait fermer.

Le vieux monde... c'est aussi le décès, en plein Paris, de Karim Ibrahim, jeune soudanais mort de faim, de froid et "de désespoir " (Libération, 11 mars) ou le suicide de Nour, Pakistanais de 17 ans (Bondy blog, 26 mars).

\section{On ne peut plus dormir tranquille lorsqu'on a ouvert les yeux une fois}

L'inquiétude monte. 30 personnalités lancent un appel en faveur des migrants dans Osons la fraternité! Les écrivains aux côtés des migrants, un ouvrage dirigé par Patrick Chamoiseau et Michel Le Bris. Mireille Delmas-Marty invite à «donner force juridique au principe d'hospitalité universel et organiser une gouvernance mondiale des mobilités humaines". Un traité pourrait "contribuer à inverser le mouvement de repli sur des sociétés de la peur qui, croyant se protéger, ne font que favoriser la fragmentation du monde " (Europe1.fr, 19 mai).
Alors que le Parlement discutait sur le (controversé) projet de loi sur l'asile et l'immigration, Daniel Cohn-Bendit et Romain Goupil en appelaient à "un nouvel ordre de l'hospitalité" (Le Monde, 17 avril). Le vieux monde se reconnaît là, dans ce projet qui "se situe dans la trop longue lignée des tentatives d'instrumentalisation politique de la question des migrants ". Les auteurs proposent la création "d'une Agence européenne de l'asile, qui aurait vocation à organiser l'instruction de la demande d'asile partout en Europe de manière indépendante des États ". Ils invitent à " penser au-delà du droit d'asile. Nous n'acceptons pas que l'on donne à croire qu'il y aurait des bons et des mauvais migrants. (...). Pour celles et ceux qui ne relèvent pas du droit d'asile (...) ce sont d'autres droits qui doivent s'ouvrir lorsqu'il y a lieu ».

Les 26 et 27 mai à Montreuil, la première assemblée plénière des États généraux des migrations adoptait un manifeste pour un « renversement $d u$ discours dominant sur la question des migrations" et « une politique migratoire fondée sur les droits fondamentaux ». II y est question d'accueil, d'un droit d'asile " effectif ", de l'égalité des droits, de la "reconnaissance des initiatives citoyennes", de liberté de circulation et d'installation... Pour mettre en œuvre cette politique, le manifeste invite à " privilégier une approche interministérielle fondée sur le droit commun"; à former "les personnels administratifs, fonctionnaires et travailleurs sociaux à la compréhension des questions migratoires, à l'interculturalité et à la logique de bienveillance » ou à faire évaluer les politiques migratoires par des autorités indépendantes.

\section{On ne tombe pas amoureux d'un taux de croissance}

"Le secteur culturel ouvre ses portes aux migrants" constate Ėve Beauvallet qui, dans Libération (16 avril), liste quelques-unes des initiatives. Ainsi, des anonymes et des bénévoles, citoyens et artistes multiplient, via le réseau des bibliothèques, les 
musées, les associations, les salles de spectacle, les librairies..., rencontres et échanges qui dessinent le concret de l'accueil culturel et cultivent le commun - parfois amoureux - quand le taux de croissance s'avachit. Nouveauté : ces initiatives sont soutenues par le ministère de la Culture. «En 2015, les réseaux français n'étaient pas encore constitués, explique Luc Gruson, responsable d'une mission d'accueil des migrants au ministère. Établissements culturels et ONG n'avaient aucune habitude de travail, il y avait une méconnaissance réciproque. Je pense aussi que les acteurs culturels attendaient un signal politique qui n'est pas venu de leur ministère à l'époque. " Pourtant, la rue de Valois est en «nonrelation " avec le ministère de l'Intérieur indique Ève Beauvallet. "Dès lors, [ces actions] apparaissent au mieux comme un combat de David contre Goliath, au pire comme un pétard mouillé tant qu'elles seront annihilées par des contre-mesures de répression d'État (...). »Comme on ne tombe amoureux ni d'un taux de croissance, ni de coups de bâton, ces mobilisations culturelles, fussentelles "symboliques", sont toujours "mieux que rien ", dixit Ariel Cypel de l'Atelier des artistes en exil.

\section{Les murs ont des oreilles. Vos oreilles ont des murs}

Mennel Ibtissem, française d'origine syrienne, arborant turban et chantant en arabe Hallelujah de Leonard Cohen, est virée de The Voice pour des tweets écrits en 2016, tweets dont elle s'est désolidarisée. Sur les réseaux, un déferlement de racisme s'est abattu sur Mathilde Edey Gamassou, parce qu'elle a été "choisie comme incarnation de Jeanne d'Arc pour les fêtes johanniques d'Orléans". Mathilde est d'origines béninoise par son père et polonaise par sa mère. "Intégré ", " assimilable ", " inassimilable ", "français ", " étranger "... Les mots barbotent dans la boue, trahissent des "imaginations malades". Et des oreilles bouchées.
Une affaire de voile a aussi défrayé la chronique : le sévère hijab de Maryam Pougetoux. La liberté de porter, ou non!, ce que certain(e)s revendiquent comme la marque d'une religion - le "voile ", c'est deux versets dans le Coran (S.33, v.59 et S.24, v.31), une recommandation plus qu'une obligation avec en prime une dispense pour les femmes... ménopausées (S.24, v.60) - est indiscutable. Le hic avec Mlle Pougetoux est qu'elle est présidente de l'Unef à Paris-IV. Sans avoir à dégainer l'artillerie lourde d'un procès en " prosélytisme ", la question se pose de savoir si, tout ou partie des membres du syndicat se reconnaissent, sans tiquer, dans l'accoutrement prétendument religieux de tel ou tel dirigeant - qui appartiendrait à telle ou telle religion d'ailleurs. On est loin de 68 et du «Comment peut-on penser librement à l'ombre d'une chapelle? ".

Plus grave. Mireille Knoll a été assassinée à Paris le 28 mars. « Il y a des préjugés qui peuvent tuer. (...) Mireille Knoll était juive, donc forcément riche aux yeux de ses agresseurs. II y en a d'autres, certes moins graves mais loin d'être anodins. Le mythe d'une communauté juive perçue comme un bloc monolithique en est un. " (The Conversation, 4 avril). Avant Mireille Knoll, il y eut Ilan Halimi, Jonathan, Gabriel et Arieh Sandler, Myriam Monsonégo, Yohan Cohen, Philippe Braham, François-Michel Saada, Yoav Hattab ou Sarah Halimi. Les murs et les préjugés enferment l'autre dans une appartenance fictive et des représentations meurtrières. Rita Hermon-Belot (École des hautes études en sciences sociales) rappelle qu'aucune communauté confessionnelle ne peut être emmurée dans des préjugés ou une institution unique. L'expression, «la communauté juive ", "n'est pas seulement impropre, elle est lourde d'effets délétères » écrit-elle. "Et justement, dans ce qui vient de se passer (...) dans notre pays apparait comme jamais qu'il n'y a pas qu'une seule voix du judaïsme en France: dans les paroles admirables de Daniel Knoll, le fils de la victime, s'opposant très nettement aux limites fixées par le président du Conseil représentatif des institutions juives de France (...). On 
peut réclamer pour les membres d'une minorité aussi le droit à la nuance et à la diversité des sensibilités, des analyses et des positions. "

Les 250 signataires du Manifeste « contre le nouvel antisémitisme " rédigé par Philippe Val lui-même (Le Parisien-Aujourd'hui, 21 avril) cèdent à une logique elle aussi «lourde d'effets délétères", en énonçant que «les Français juifs ont 25 fois plus de risques d'être agressés que leurs concitoyens musulmans ». Ils évoquent "une épuration ethnique " menée par «la terreur islamiste en France ", faisant même de l'antisionisme le cache-sexe d'un "nouvel antisémitisme». Ce constat posé, ils demandent "que les versets du Coran appelant au meurtre et au châtiment des juifs, des chrétiens et des incroyants soient frappés d'obsolescence par les autorités théologiques (...) afin qu'aucun croyant ne puisse s'appuyer sur un texte sacré pour commettre un crime. (...) Avant que la France ne soit plus la France". Bientôt, reviendra, "la France aux Français ". Pour Mohamed Arkoun la question n'était pas de savoir ce que dit le Coran mais "Qu'est-ce que je lis ? " et "Quifait autorité ? ", rappelant que " toute interprétation est sujette à débat même si l'on dispose d'une autorité religieuse quiest infiniment respectée ». "Tout ce qui est discutable est à discuter", proclamait un autre slogan de Mai 68. 